\title{
Acute pancreatitis: update on management
}

\begin{abstract}
As acute
pancreatitis

is common,

costly and

potentially life-

threatening, it

is important

that

management

A cute pancreatitis is a common acute surgical condition. The annual incidence worldwide is 4.9-73.4 cases per 100000 people, ${ }^{1,2}$ with the incidence in Australia on the higher end of the spectrum. ${ }^{3}$ The mortality rate for pancreatitis is between $1.5 \%$ and $4.2 \%$ in large epidemiological studies, ${ }^{4-6}$ but varies according to the severity of pancreatitis, increasing to $30 \%$ in those with infected pancreatic necrosis. ${ }^{7}$ A recent Australian study has reported a low mortality rate of $0.08 \%,{ }^{8}$ thought to be due to earlier recognition of severe pancreatitis and the appropriate use of intensive care support. ${ }^{8}$
\end{abstract}

is guided by

an evidence-

based

approach

Maryam Nesvaderani
MBBS, BSC(Hons)

Guy DEslick

DrPH, PhD, FACE

Michael R Cox

MBBS, MS, FRACS

1 University of Sydney,

Sydney, NSW.

2 Nepean Hospital

Sydney, NSW.

eslickg@

med.usyd.edu.au

doi: 10.5694/mjal4.01333

As acute pancreatitis is common, costly and potentially life-threatening, it is important that management is guided by an evidence-based approach. The American College of Gastroenterology (ACG) and the International Association of Pancreatology (IAP) in collaboration with the American Pancreatic Association (APA) have recently published new guidelines for the management of acute pancreatitis. ${ }^{910}$ These guidelines reviewed all of the evidence of a wide range of areas within the management of acute pancreatitis and made recommendations of various strengths based on the level of available evidence.

Here, we provide an update on diagnosing and managing acute pancreatitis based on the strong recommendations in those guidelines. These recommendations are summarised in Box 1.

There are two aspects of management that have changed in the newer 2013 guidelines. The most significant change is the recommendation that patients with biliary pancreatitis and concurrent cholangitis or common bile duct obstruction should have early endoscopic retrograde cholangiopancreatography (ERCP), whereas the old guidelines recommended ERCP for all patients with severe pancreatitis. This is based on new evidence from a meta-analysis showing no benefit of ERCP in severe pancreatitis. Second, the definition of severe pancreatitis has been revised in the new guidelines to include moderately severe acute pancreatitis, distinct from severe pancreatitis, in accordance with the revision of the Atlanta classification published in 2013.?

\section{Aetiology of pancreatitis}

Gallstones and alcohol together make up to $80 \%$ of all causes of pancreatitis, ${ }^{11}$ with gallstones leading at a ratio of $2: 1$ in a recent Australian study. ${ }^{12}$ The incidence of idiopathic pancreatitis is increasing, ${ }^{13}$ which may be explained by increasing rates of morbid obesity in our communities. One explanation may be an increase in biliary microlithiasis given that most cases of idiopathic pancreatitis are actually biliary pancreatitis due to microlithiasis. ${ }^{14}$
Summary

- Acute pancreatitis is a common acute surgical condition associated with high morbidity and mortality in severe cases.

- New guidelines for management have recently been published by the American College of Gastroenterology and by the International Association of Pancreatology in collaboration with the American Pancreatic Association.

- The main differences between the new and previous versions of the guidelines relate to the use of endoscopic retrograde cholangiopancreatography (ERCP) and the addition of the new severity category of 'moderately severe acute pancreatitis'

- All patients with pancreatitis should have its cause determined by features of the history, results of laboratory tests (liver function tests, serum calcium triglyceride levels) and findings on transabdominal ultrasound. Those with idiopathic pancreatitis should have endoscopic ultrasound as a first-line investigation.

- Acute pancreatitis should be managed with aggressive hydration with intravenous fluids and fasting.

- Oral feeding can be recommenced in mild pancreatitis once pain and nausea and vomiting have resolved.

- Patients with mild biliary pancreatitis should have a laparoscopic cholecystectomy during their index admission.

- In addition to aggressive intravenous fluid resuscitation and fasting, patients with severe pancreatitis should have enteral feeding (nasoenteric or nasogastric feeds) commenced 48 hours after presentation. Total parenteral nutrition should be avoided where possible.

- All patients with organ failure or severe pancreatitis as defined by the revised version of the Atlanta classification should be managed in an intensive care setting.

- Patients with biliary pancreatitis and concurrent cholangitis should have endoscopic retrograde cholangiopancreatography within 24 hours of presentation.

\section{Diagnosis}

Diagnosis is established by the presence of two of the three criteria:

- acute upper abdominal pain;

- serum amylase and/or lipase levels greater than three times the upper limit of normal; and/or

- characteristic findings from abdominal imaging; either computed tomography (CT), magnetic resonance imaging (MRI) or ultrasound., ${ }^{7,10}$ 


\section{Summary of recommendations}

Diagnosis

The diagnosis of acute pancreatitis is made if two of the following three criteria are met:

(i) upper abdominal pain;

(ii) serum amylase and/or lipase levels > 3 times the upper limit of normal;

(iii) characteristic findings on abdominal imaging

\section{Use of computed tomography (CT)}

CT of the abdomen should be performed if either: (i) there is an unclear diagnosis; or

(ii) the patient's condition fails to improve clinically after 3-5 days.

\section{Aetiology}

For all patients with acute pancreatitis, aetiology should be determined by features of the history, results of laboratory tests (liver function tests, serum calcium triglyceride levels) and findings on transabdominal ultrasound.

Patients with idiopathic pancreatitis should have endoscopic ultrasound \pm magnetic resonance cholangiopancreatography to assess for microlithiasis, neoplasms and chronic pancreatitis.

\section{Severity}

Acute pancreatitis should be predicted on the basis of patient characteristics (age, comorbid conditions, body mass index, presence of systemic inflammatory response syndrome, and laboratory findings. There is no evidence that a particular severity scoring system is more accurate than any other.

\section{Management of mild pancreatitis}

Early aggressive intravenous hydration with isotonic crystalloid solution within the first 12-24 hours.

Oral feeding can be commenced once pain is resolving and there is no nausea or vomiting.

Cholecystectomy in mild biliary pancreatitis should be performed during the index admission.

\section{Management of severe pancreatitis}

Early aggressive intravenous hydration with isotonic crystalloid solution within the first 12-24 hours.

Patients with severe acute pancreatitis as defined by the revised Atlanta classification (persistent organ failure) ${ }^{7}$ should be managed in the intensive care setting.

In severe acute pancreatitis, enteral feeding should be commenced early in patients requiring nutritional support.

Endoscopic retrograde cholangiopancreatography (ERCP)

ERCP should be performed within 24 hours of presentation in patients with concurrent acute cholangitis.

\section{Antibiotics}

Routine, prophylactic intravenously delivered antibiotics are not recommended in acute pancreatitis.

$\mathrm{IA}=$ strong recommendation from both guidelines, high quality of evidence. $1 \mathrm{~B}=$ strong recommendation from both guidelines, moderate quality of evidence. $1 \mathrm{C}=\mathrm{strong}$ recommendation from both guidelines, low quality of evidence. $2 \mathrm{~B}=$ Weak recommendation from both guidelines, moderate quality of evidence. $2 \mathrm{C}=$ Weak recommendation from both guidelines, low quality of evidence.

*Quality of evidence was adapted from the assessment of evidence in the guidelines of the American College of Gastroenterology and the International Association of

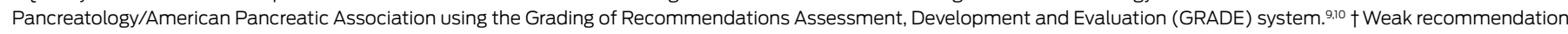
with no consensus between guidelines, low quality of evidence.

CT should only be used for initial assessment if there is doubt about the clinical diagnosis or a normal level of lipase and/or amylase because of a delayed presentation. ${ }^{910}$ The doubt in clinical diagnosis may be because the pain is not typical or an alternative diagnosis needs to be excluded (eg, if there is a suspicion of bowel perforation or ischaemia).

Routine use of CT scanning is not recommended in patients with a clear diagnosis of pancreatitis established by the presence of the first two criteria. CT scanning should be reserved for those whose condition fails to improve clinically after 5 days to assess the severity and extent of necrosis, as it is after this time interval that the presence and extent of pancreatic necrosis are most obvious (Box 2). ${ }^{9,10}$ The reason for not performing routine early $\mathrm{CT}$ scans is based on strong evidence that they do not improve clinical outcomes, increase the risk of contrast allergy and nephrotoxicity, and increase the duration of hospital stay. ${ }^{15,16}$

\section{Assessment of aetiology}

Determining the aetiology of pancreatitis is important for guiding management and preventing recurrence. The following should be performed in all patients presenting with acute pancreatitis:

- a detailed history, including alcohol intake, medications, hyperlipidaemia, trauma, recent ERCP, and family history of pancreatitis;

- abdominal ultrasound to evaluate for cholelithiasis and 
2 Arterial phase computed tomography (CT) images (Day 0,* Day $4^{\dagger}$ and Day $7^{\ddagger}$ ) of a 65-year-old man with severe idiopathic pancreatitis who developed pancreatic necrosis
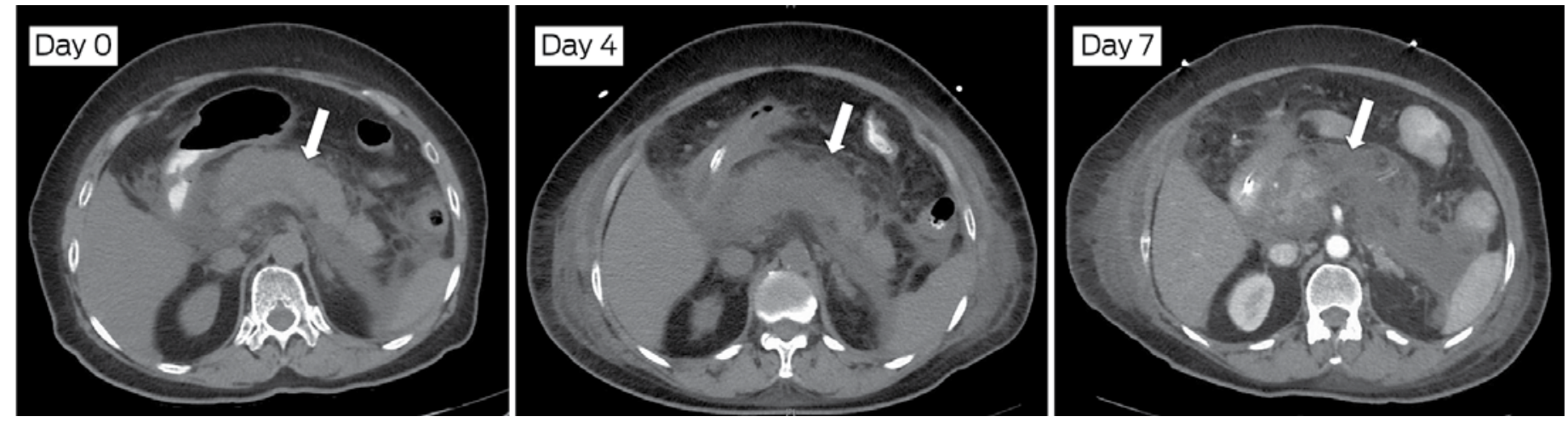

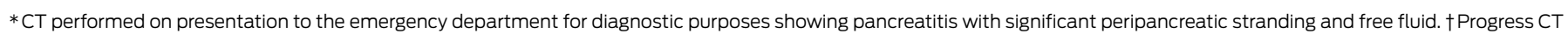

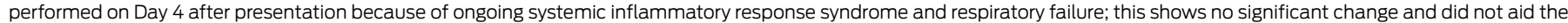
management. $\ddagger$ Another progress CT, performed on Day 7 after presentation because of worsening sepsis; this shows pancreatic necrosis.

Arrows indicate the pancreas in each image.

- laboratory tests for liver enzyme, serum triglyceride and calcium levels. ${ }^{9,10}$

Findings on liver function tests, particularly the level of alanine aminotransferase (ALT), can be used as a surrogate marker for gallstone pancreatitis. ALT levels greater than or equal to $150 \mathrm{IU} / \mathrm{L}$ have a positive predictive value of $95 \%$ in diagnosing gallstone pancreatitis. ${ }^{17}$

The patient is considered to have "idiopathic pancreatitis" if the above fails to identify a cause. Endoscopic ultrasound is recommended as a first-line investigation in those with idiopathic pancreatitis to assess for occult microlithiasis, neoplasms and chronic pancreatitis. ${ }^{10}$ If findings on endoscopic ultrasound are negative, magnetic resonance cholangiopancreatography (MRCP) should be performed as a second-line investigation. ${ }^{10} \mathrm{~A}$ recent study has shown that endoscopic ultrasound and MRCP can identify a possible biliary cause in $57 \%$ of cases of "idiopathic" pancreatitis. ${ }^{14}$ However, it should be noted that there is a low level of evidence to support the use of endoscopic ultrasound as a first-line investigation, and the guidelines advise that the relative risks and benefits of endoscopic investigation in these patients are unclear.

\section{Assessment of severity}

Severity is an important indicator of mortality and facilitates management decisions about the need for a critical care bed and nutritional support. The revised Atlanta classification ${ }^{7}$ is used to define severity of pancreatitis as follows.

\section{Mild acute pancreatitis}

- No organ failure or local or systemic complications.

- Most episodes of pancreatitis are mild and self-limiting, lasting less than 7 days.

\section{Moderately severe acute pancreatitis}

- Transient organ failure of less than 48 hours or local complications (peripancreatic fluid collection, pancreatic necrosis) or systemic complications (exacerbation of pre-existing disease).

\section{Severe acute pancreatitis}

- Persistent organ failure of greater than 48 hours.

- High mortality rate of $20 \%-30 \%$.

There are several scoring systems available to predict which patients will develop severe disease, including the Acute Physiology and Chronic Health Evaluation II (APACHE II), ${ }^{18}$ Ranson criteria ${ }^{19}$ and modified Glasgow scores, ${ }^{20}$ which rely on clinical and laboratory values, and the Balthazar score, which is based on CT findings. ${ }^{21}$ A newer prognostic scoring system, the Bedside Index for Severity in Acute Pancreatitis (BISAP), relies on the blood urea nitrogen level, impaired mental status, systemic inflammatory response syndrome (SIRS), age over 60 years and pleural effusions to stratify patients, and has a prognostic accuracy similar to the other scoring systems. ${ }^{22}$ There is no definitive consensus as to which scoring system should be used, and recent guidelines suggest that existing scoring systems have limited value as they all have a good negative predictive value but low positive predictive value., ${ }^{9,23}$ However, it is recommended that risk assessment be performed for all patients with pancreatitis to stratify them into higher and lower risk categories. ${ }^{9}$ Most scoring systems require 48 hours for accurate scoring, and CT-based systems are also inaccurate in the early course of disease as necrosis only becomes evident after 48 hours. ${ }^{9}$ Both the ACG and IAP/APA guidelines recommend a clinical approach to predicting the outcome of acute pancreatitis, combining patient factors (age, body mass index, comorbid disease), the presence of SIRS and laboratory findings (elevated creatinine level, rising haematocrit). ${ }^{9,10}$ 


\section{Mild pancreatitis}

Early aggressive intravenous hydration (with isotonic crystalloid solution, such as Hartmann's solution) in the first 12-24 hours of presentation is essential, with frequent reassessment of fluid status. ${ }^{9,10}$ Early aggressive fluid resuscitation reduces the risk and the extent of pancreatic necrosis, leading to improved clinical outcomes. The success of this therapy is thought to be related to improved pancreatic perfusion. In addition, patients should be kept nil by mouth until abdominal pain and nausea and vomiting are resolving. ${ }^{9,10}$ In mild pancreatitis, nutritional support is rarely needed as oral feeding is usually commenced early.

In patients with mild biliary pancreatitis, laparoscopic cholecystectomy should be performed during the index admission. ${ }^{9,10}$ A delay of more than 4 weeks is associated with recurrent gallstone pancreatitis or other complications of gallstone disease. ${ }^{24}$

\section{Severe pancreatitis}

In addition to aggressive intravenous fluid resuscitation and fasting, patients with severe pancreatitis may require an intensive care bed and nutritional support. All patients with severe acute pancreatitis as defined by the revised Atlanta classification ${ }^{7}$ (persistent organ failure) should be treated in an intensive care setting., ${ }^{910}$

Enteral feeding is indicated in patients with predicted severe acute pancreatitis and should be commenced early, ideally after 48 hours of fasting., ${ }^{9,10}$ Early nutritional support with nasoenteric or nasogastric feeding has been shown to improve clinical outcomes in patients with severe pancreatitis, resulting in reduced infective complications, a reduced length of stay and a tendency toward improved mortality. ${ }^{25,26}$ Parenteral nutrition should be avoided because of the increased risk of infectious complications. ${ }^{10,11}$

Previous guidelines from 2005 recommended that all patients with severe biliary pancreatitis should have ERCP performed within 72 hours of presentation. ${ }^{27}$ However, a recent meta-analysis has shown that early ERCP does not affect mortality or complications in severe gallstone pancreatitis, and a benefit is only seen in patients with concurrent cholangitis or biliary obstruction. ${ }^{28}$ The more recent guidelines reflect this finding, with both the IAP/APA and ACG guidelines recommending that ERCP should be performed at less than 24 hours after presentation in patients with concurrent acute cholangitis rather than in all patients with severe biliary pancreatitis.,10

Routine antibiotic therapy is not indicated in severe acute pancreatitis. ${ }^{910}$ Antibiotics should only be given for extrapancreatic infection (eg, cholangitis) or to patients with infected pancreatic necrosis. Meta-analyses have not shown improvement in outcomes with prophylactic antibiotic therapy, ${ }^{29,30}$ and some studies show an increased risk of pancreatic fungal infection with routine antibiotic use. $^{31,32}$

\section{Transfer to specialist centres}

Referral to a specialist centre is recommended for patients with severe acute pancreatitis and those who may require surgical or endoscopic intervention. ${ }^{10} \mathrm{~A}$ specialist centre is defined as a high-volume centre with intensive care facilities and daily access to interventional radiology, endoscopy and surgical services. There is evidence that management of severe pancreatitis in high-volume centres results in shorter length of stay and lower mortality rates. ${ }^{33}$

Competing interests: No relevant disclosures.

Provenance: Not commissioned; externally peer reviewed.

References are available online at www.mja.com.au. 
1 Fagenholz PJ, Castillo CF, Harris NS, et al. Increasing United States hospital admissions for acute pancreatitis, 1988-2003. Ann Epidemiol 2007; 17: 491-497.

2 Yadav D, Lowenfels AB. Trends in the epidemiology of the first attack of acute pancreatitis: a systematic review. Pancreas 2006; 33: 323-330.

3 Australian Institute of Health and Welfare. The burden of disease and injury in Australia 2003. Canberra: AlHW, 2007. (AlHW Cat. No. PHE 82.) http://www.aihw.gov.au/publicationdetail/?id=6442467990 (accessed 4 Feb 2015).

4 Frey $\mathrm{CF}$, Zhou H, Harvey DJ, White $\mathrm{RH}$. The incidence and case-fatality rates of acute biliary, alcoholic, and idiopathic pancreatitis in California, 1994-2001. Pancreas 2006; 33: 336-344.

5 Chen Y, Zak Y, Hernandez-Boussard T, et al. The epidemiology of idiopathic acute pancreatitis, analysis of the nationwide inpatient sample from 1998 to 2007. Pancreas 2013; 42: 1-5.

6 Appelros S, Borgström A. Incidence, aetiology and mortality rate of acute pancreatitis over 10 years in a defined urban population in Sweden. Br J Surg 1999; 86: 465-470.

7 Banks PA, Bollen TL, Dervenis C, et al; Acute Pancreatitis Classification Working Group. Classification of acute pancreatitis--2012: revision of the Atlanta classification and definitions by international consensus. Gut 2013; 62: 102-111.

8 Jacob AO, Stewart P, Jacob O. Early surgical intervention in severe acute pancreatitis: Central Australian experience. ANZ J Surg 2014. 30 May 2014. doi: 10.1111/ans.12707. [Epub ahead of print].

9 Tenner S, Baillie J, Dewitt J, et al. American College of Gastroenterology guideline: management of acute pancreatitis. Am J Gastroenterol 2013; 108: 1400-1415.

10 Working Group IAP/APA Acute Pancreatitis Guidelines. IAP/ APA evidence-based guidelines for the management of acute pancreatitis. Pancreatology 2013; 13(4 Suppl 2): el-el5.

1 Kingsnorth A, O'Reilly D. Acute pancreatitis. BMJ 2006; 332: 1072-1076.

12 Barreto SG, Tiong L, Williams R. Drug-induced acute pancreatitis in a cohort of 328 patients. A single-centre experience from Australia. JOP 2011; 12: 581-585.

13 Lee JK, Enns R. Review of idiopathic pancreatitis. World J Gastroenterol 2007; 13: 6296-6313.

14 Ortega AR, Gómez-Rodríguez R, Romero M, et al. Prospective comparison of endoscopic ultrasonography and magnetic resonance cholangiopancreatography in the etiological diagnosis of "idiopathic" acute pancreatitis. Pancreas 2011; 40: 289-294.

15 Fleszler F, Friedenberg F, Krevsky B, et al. Abdominal computed tomography prolongs length of stay and is frequently unnecessary in the evaluation of acute pancreatitis. Am J Med Sci 2003; 325: 251-255.

16 Spanier BW, Nio Y, van der Hulst RW, et al. Practice and yield of early CT scan in acute pancreatitis: a Dutch Observational Multicenter Study. Pancreatology 2010; 10: 222-228.

17 Tenner S, Dubner H, Steinberg W. Predicting gallstone pancreatitis with laboratory parameters: a meta-analysis. Am J Gastroenterol 1994; 89: 1863-1866.

18 Knaus WA, Draper EA, Wagner DP, Zimmerman JE. APACHE II: a severity of disease classification system. Crit Care Med 1985; 13: 818-829.
19 Ranson JH, Rifkind KM, Turner JW. Prognostic signs and nonoperative peritoneal lavage in acute pancreatitis. Surg Gynecol Obstet 1976; 143: 209-219.

20 Blamey SL, Imrie CW, O'Neill J, et al. Prognostic factors in acute pancreatitis. Gut 1984; 25: 1340-1346.

21 Balthazar EJ, Robinson DL, Megibow AJ, Ranson JH. Acute pancreatitis: value of CT in establishing prognosis. Radiology 1990; 174: 331-336.

22 Park JY, Jeon TJ, Ha TH, et al. Bedside index for severity in acute pancreatitis: comparison with other scoring systems in predicting severity and organ failure. Hepatobiliary Pancreat Dis Int 2013; 12: 645-650.

23 Phillip V, Steiner JM, Algül H. Early phase of acute pancreatitis: assessment and management. World J Gastrointest Pathophysiol 2014; 5: 158-168.

24 van Baal MC, Besselink MG, Bakker OJ, et al. Timing of cholecystectomy after mild biliary pancreatitis: a systematic review. Ann Surg 2012; 255: 860-866.

25 Sun JK, Mu XW, Li WQ, et al. Effects of early enteral nutrition on immune function of severe acute pancreatitis patients. World J Gastroenterol 2013; 19: 917-922.

26 McClave SA, Chang WK, Dhaliwal R, Heyland DK. Nutrition support in acute pancreatitis: a systematic review of the literature. JPEN J Parenter Enteral Nutr 2006; 30: 143-156.

27 Working Party of the British Society of Gastroenterology; Association of Surgeons of Great Britain and Ireland; Pancreatic Society of Great Britain and Ireland; Association of Upper GI Surgeons of Great Britain and Ireland. UK guidelines for the management of acute pancreatitis. Gut 2005; 54 Suppl 3: iiil-iiig.

28 Tse F, Yuan Y. Early routine endoscopic retrograde cholangiopancreatography strategy versus early conservative management strategy in acute gallstone pancreatitis. Cochrane Database Syst Rev 2012; (5): CD009779.

29 Wittau M, Mayer B, Scheele J, et al. Systematic review and meta-analysis of antibiotic prophylaxis in severe acute pancreatitis. Scand J Gastroenterol 2011; 46: 261-270.

30 Villatoro E, Mulla M, Larvin M. Antibiotic therapy for prophylaxis against infection of pancreatic necrosis in acute pancreatitis. Cochrane Database Syst Rev 2010; (5): CD002941.

31 Kochhar R, Ahammed SK, Chakrabarti A, et al. Prevalence and outcome of fungal infection in patients with severe acute pancreatitis. J Gastroenterol Hepatol 2009; 24: 743-747.

32 Isenmann R, Schwarz M, Rau B, et al. Characteristics of infection with Candida species in patients with necrotizing pancreatitis. World J Surg 2002; 26: 372-376.

33 Singla A, Simons J, Li Y, et al. Admission volume determines outcome for patients with acute pancreatitis. Gastroenterology 2009; 137: 1995-2001. 